\title{
Eurostudia
}

\section{La crise économique comme architecte de l'espace public européen. Le cas des élections européennes françaises de 2014}

\section{Capucine Berdah et Kim Désert}

Volume 12, numéro 1, 2017

L’Union européenne et le sentiment d'appartenance

The European Union and the Sense of Belonging

Die Europäische Union und das Zugehörigkeitsgefühl

URI : https://id.erudit.org/iderudit/1039779ar

DOI : https://doi.org/10.7202/1039779ar

Aller au sommaire du numéro

Éditeur(s)

Le Centre canadien d'études allemandes et européennes

ISSN

1718-8946 (numérique)

Découvrir la revue

Citer cet article

Berdah, C. \& Désert, K. (2017). La crise économique comme architecte de

l'espace public européen. Le cas des élections européennes françaises de 2014.

Eurostudia, 12(1), 27-45. https://doi.org/10.7202/1039779ar
Résumé de l'article

En donnant plus de visibilité et un rôle politique accru à l'Union européenne, la crise permet de structurer un espace public au niveau européen. Une des façons de vérifier cette hypothèse est d'étudier le discours médiatique. Cet article analyse l'impact de la crise sur le discours médiatique français. Dans une démarche exploratoire, nous comparons les campagnes électorales des élections européennes de 2009 (avant la crise) et de 2014 (pendant la crise) afin d'observer s'il y a une évolution dans le traitement de l'information. Nos données suggèrent que la crise a un impact sur la visibilité de l'Union européenne en créant un espace médiatique et politique qui lui est propre, et qu'elle n'avait pas en 2009.
Tous droits réservés ( Le Centre canadien d'études allemandes et européennes, 2017
Ce document est protégé par la loi sur le droit d'auteur. L'utilisation des services d’Érudit (y compris la reproduction) est assujettie à sa politique d'utilisation que vous pouvez consulter en ligne.

https://apropos.erudit.org/fr/usagers/politique-dutilisation/ 


\section{Capucine Berdah}

Université de Montréal

\section{Kim Désert}

Université de Montréal

\section{Résumé}

En donnant plus de visibilité et un rôle politique accru à l'Union européenne, la crise permet de structurer un espace public au niveau européen. Une des façons de vérifier cette hypothèse est d'étudier le discours médiatique. Cet article analyse l'impact de la crise sur le discours médiatique français. Dans une démarche exploratoire, nous comparons les campagnes électorales des élections européennes de 2009 (avant la crise) et de 2014 (pendant la crise) afin d'observer s'il y a une évolution dans le traitement de l'information. Nos données suggèrent que la crise a un impact sur la visibilité de l'Union européenne en créant un espace médiatique et politique qui lui est propre, et qu'elle n'avait pas en 2009.

\section{Introduction}

Depuis 2009, l'Union européenne (UE) connaît une crise économique à laquelle elle peine à faire face. Le 5 novembre 2009, le gouvernement grec, alors récemment élu, annonce un déficit de $12,7 \%$, plongeant ainsi le pays dans une crise économique sans précédent (Liberti 2012 : 19). La crise de la dette s'étend à d'autres membres de la zone euro : le Portugal, l'Irlande, l'Espagne et l'Italie. En l'absence de gouvernement économique, de budget et d'instruments de gestion de crise communs, l'UE entre alors dans une phase de crise politique. Les débats s'orientent sur les sorties de crise possibles, sur le déficit démocratique latent et sur la légitimité de l’UE.

$\mathrm{Au}$ lendemain de l'annonce du déficit grec, l'exécutif européen a immédiatement pris un certain nombre de mesures afin de contrôler la contagion 
de la crise économique à d'autres pays de la zone euro. Il fallait rapidement mettre en œuvre des plans de sauvetage afin que les pays touchés puissent honorer leurs dettes. Toutefois, l'octroi de ces aides financières a été associé à des conditionnalités: coupures budgétaires, hausse des taxes, libéralisation de l'économie et réforme des retraites, par exemple.

Dans cet article, nous considérons la crise économique comme une fenêtre d'opportunité permettant de structurer un espace de débat sur des enjeux européens à l'échelle nationale. Comme elle politise (Baglioni et Hurrelmann 2014), qu'elle médiatise (Picard 2015) et qu'elle crée des mouvements de contestation (Albo, Panitch et Chibber 2013), la crise permet l'émergence d'espaces de communication donnant une nouvelle visibilité à l'UE (Meijers 2013). Notre objectif est de vérifier l'existence d'un lien entre la crise économique et l'européanisation de l'espace public en posant la question de recherche suivante: le contexte de crise économique structure-t-il un espace public européen en France? Afin de répondre à cette question, nous analysons la couverture médiatique française lors des élections européennes de 2009 et 2014. Considérées comme des manifestations de l'espace public, les élections européennes nous permettent de mieux comprendre l'impact de la crise sur cet espace. À travers une analyse de contenu médiatique comparée (2009 et 2014), nous explorons l'hypothèse suivante: en donnant plus de visibilité et un rôle politique accru à l'UE, la crise permet d'européaniser l'espace public français. Dans une démarche exploratoire, notre article vise à apporter de nouveaux éléments empiriques au débat sur l'espace public européen.

\section{Cadre théorique}

\section{L'espace public européen}

Les recherches sur l'espace public européen (EPE) émergent dès les années 1990, en parallèle des débats sur le déficit démocratique, et s'articulent autour de l'interprétation et de la valeur de la notion d'EPE. D'un côté, l'intérêt heuristique de ce concept est remis en cause, car comme il a été élaboré dans un cadre national, il ne se prête pas à un changement d'échelle. D'un autre côté, l'approche exclusivement politique généralement associée à la notion d'espace public a elle aussi été critiquée: comme elle serait trop proche de l'«agora» grecque, il 
s'agirait de lui donner une dynamique plus contemporaine (Desroches et Innerarity 2011). De manière générale, la communication reste le dénominateur commun (Bärenreuter et al. 2009), ce qui nous permet de donner à l'EPE la définition suivante: "an intersubjectively shared, communicatively constructed system of mutual observance without a concrete social order or membership and therefore applicable to contingent situations » (Ibid: 10).

Même si cette définition tend à unifier la communauté scientifique autour d'une compréhension unique, elle ne réconcilie pas les divergences qui subsistent autour de l'échelle à adopter. Deux écoles de pensée se dessinent. D'une part, il s'agit de concevoir l'EPE comme un objet transnational, transcendant les frontières, autonome et alimenté par les espaces publics nationaux. Selon la définition donnée par Habermas de l'espace public, il s'agit « [ $\left.\mathrm{d}^{\prime}\right]$ un processus au cours duquel le public constitué d'individus faisant usage de leur raison s'approprie la sphère publique contrôlée par l'autorité et la transforme en une sphère où la critique s'exerce contre le pouvoir de l'état » (Habermas 1978 : 61). Dans la droite lignée d'une conception fédéraliste, il est ici question de transposer les dynamiques fédérales sur celles de l'UE (Weisbein 2002: 91-108). Les institutions européennes sont alors érigées en tant qu'État et l'espace public serait un espace de médiation entre ce dernier et la sphère privée, où les citoyens délibèreraient publiquement des questions politiques. Dans ce modèle, l'EPE serait une réplique du système national au niveau européen. Cependant, comme l'UE n'est pas une fédération, et ne tend pas à l'être (Hix 1999: 33), il devient difficile d'affirmer l'effectivité d'une réalité empirique, même naissante, d'un tel espace. De plus, chaque concept auquel touche cet espace fait débat dans la sphère scientifique. Qu'il s'agisse de "peuple européen», de "citoyenneté européenne », ou de "démocratie européenne », les débats sont houleux autour de la justification de leur réalité empirique (Dacheux 2003 : 19).

D'autre part, venue des études médiatiques en communication, une seconde école préfère parler d'une européanisation des espaces publics nationaux (Koopmans et Statham 2010). Dans «The Many Faces of Europeanization », Olsen définit l'européanisation comme un développement politique faisant de l'Europe une entité plus distincte, plus cohérente et plus forte (Olsen 2002: 13). À cet égard, il avance que " the development of a European sphere for public will and opinion 
formation contributes to common conceptions of legitimate political organization and governance and a shared feeling of belonging, giving direction to collective action capabilities» (Olsen 2002 : 940). Une sphère publique européanisée est alors susceptible d'émerger quand les mêmes sujets sont abordés en même temps et avec la même pertinence dans différents espaces publics (Trenz et Eder 2004 : 20). Deux niveaux d'européanisation sont alors identifiés: soit par un échange intensifié entre les différentes sphères publiques nationales (européanisation horizontale), soit par l'importation d'acteurs et de questions européennes dans les sphères publiques nationales (européanisation verticale) (Koopmans et Statham 2010). À cette européanisation, Virginie Guiraudon voit plusieurs raisons : "Soit parce que des acteurs européens interviennent, soit parce que des acteurs nationaux interpellent les institutions européennes ou se mobilisent au niveau européen, soit parce qu'un cadrage européen est utilisé pour justifier une position. » (Guiraudon 2002 : 9)

\section{Crise, médias et européanisation de l'espace public national}

Crise de la zone euro, crise politique, crise identitaire, etc. : la crise revêt tant de facettes qu'il est parfois difficile d'en saisir pleinement le sens. Même s'il existe un grand nombre de définitions, dans le cadre de cet article, nous avons choisi de retenir celle de Julien Freund : "la crise est une situation collective caractérisée par des contradictions et ruptures, grosses de tensions et de désaccords, qui rendent les individus et les groupes hésitants sur la ligne de conduite à tenir, parce que les règles et les institutions ordinaires restent en retrait ou sont même parfois déphasées par rapport aux possibilités nouvelles qu'offrent les intérêts et les idées qui surgissent du changement, sans que l'on puisse cependant se prononcer clairement sur la justesse et l'efficacité des voies nouvelles » (Freund 1976 : 103).

Selon Freund, l'impact majeur de la crise est la création de conflits : en contexte de crise économique, les gouvernements répondent par des politiques de sortie de crise guidées par des idéologies spécifiques. Toutefois, lors de la crise de 2010, les gouvernements nationaux européens n'avaient aucune compétence exécutive pour sa résolution, la politique monétaire étant une compétence exclusive de l'UE et les budgets nationaux étant contraints par le Pacte de stabilité et de croissance. Ainsi, le conflit s'est déplacé du niveau national au 
niveau européen à cause du transfert de compétences. La politique d'austérité a primé toutes les autres options de sortie de crise : «it is equally reflecting a dogmatic view in which fiscal policy is incapable of demand management and the scope of public administrations has to be fettered and limited. This ideology has led member states to implement massive fiscal austerity (OFCE 2012 : 7).

La crise économique, en focalisant l'attention sur la responsabilité de l'UE dans la résolution du problème, démarre selon nous le processus d'européanisation de l'espace public national. Comme la visibilité médiatique conditionne l'émergence de la littérature (Pfetsch 2004 : 6), nous considérons les médias comme un facteur contribuant au processus d'européanisation de la sphère publique nationale (Peter et De Vreese 2004 : 4). Selon les théories de l'espace public, les médias de masse constituent un forum institutionnalisé de débat, servant de lien entre le public et la structure institutionnelle. Dans cette fonction, ils sont des relais d'information entre les différents problèmes et les différents acteurs de la sphère politique. Cependant, le rôle des médias n'est pas simplement de servir de relais de communication ou de forum d'échange: ils doivent être également considérés comme des acteurs politiques à part entière de l'espace public qui apportent leur propre voix. Plus particulièrement, ils y arrivent en mettant en lumière certains débats publics et en exprimant leurs propres opinions (Pfetsch $2004: 5$ ).

Depuis le début des années 1990, un certain nombre de chercheurs ont constaté que la communication entre l'UE et le public dépend, de façon cruciale, des médias: selon les données de l'Eurobaromètre de 2003, les deux tiers des citoyens de l'UE identifient systématiquement les médias comme la plus importante source d'information politique (Peter et De Vreese 2004 : 4). Comme la crise touche des domaines de compétences exclusifs ou partagés, l'UE est propulsée comme acteur de premier plan et focalise l'attention des médias :

European news coverage since the emergence of the crisis in 2008 has played a central role in shaping public perceptions of the crisis and public reactions to the responses of policy makers. It has created highly mediated portrayals of Europe, European institutions, EU members and the euro, and Europeanness itself. The lenses provided by news organizations across Europe affected public understanding of the developments, their causes, the responsibilities for addressing the crisis, and the roles and effectiveness of 
32 L'Union européenne et le sentiment d'appartenance

European institutions, and these portrayals have significant implications for European identity and integration. (Picard $2015: 1$ )

Cette visibilité progressive de l'UE est rendue possible par une publicisation de ses politiques de résolution de crise dans les médias: ceux-ci deviennent un intermédiaire incontournable entre la structure institutionnelle européenne et l'EPE. L'espace médiatique, en d'autres termes, devient une arène de représentation et de manifestation de l'espace public.

\section{Méthodologie}

Dans cet article, nous combinons une analyse comparative diachronique à une analyse de contenu. En nous concentrant sur le cas de la France, nous avons choisi de comparer la campagne des élections européennes de 2009 et 2014 dans une perspective de most similar system design. Comme notre objectif est d'observer l'impact de la crise économique sur l'européanisation de l'espace français, nous avons choisi deux contextes similaires à des points temporels différents, le premier avant la crise, le second pendant la crise.

\section{Les élections européennes comme "moments européens »}

Dans le cadre de campagnes électorales, la sphère médiatique constitue le principal espace de communication entre les candidats et les électeurs. Elle est souvent perçue comme jouant un rôle primordial dans le processus démocratique (Brack, Rittelmeyer et Stanculescu 2010: 183). Parce que les messages de campagne parviennent essentiellement aux électeurs par ce biais (Trenz et Eder 2004), une des fonctions principales de la sphère médiatique est de lier le citoyen avec la sphère politique: "most voters [...] experience politics primarily through the media » (de Vreese, Banducci, Semetko et Boomgaarden 2006 : 492). Ce rôle de médiation est renforcé dans le cas d'institutions distantes des citoyens, comme le Parlement européen, car ceux-ci n'en possèdent, bien souvent, qu'une connaissance ou expérience limitée: "citizens across Europe rely on the media for information about the European Union and European integration» (de Vreese 2001 : 284). Pour cette raison, les élections européennes sont des moments $t$ qui incarnent l'EPE: elles permettent une apparition tangible de cet espace en lui créant une arène médiatique propre. 
Pour ces deux élections, nous conduisons l'analyse pendant les deux semaines de campagne électorale officielle et les deux semaines suivant le jour $\mathrm{du}$ scrutin. Pour les élections de 2009, nous avons étudié les semaines du 25 mai au 21 juin et, pour 2014, les semaines du 12 mai au 8 juin.

\section{Analyse de contenu médiatique}

À l'aide du logiciel d'analyse de texte Wordstat, nous avons mené une analyse thématique sur deux quotidiens grand format à grande diffusion d'appartenance politique opposée : Le Monde et Le Figaro.

Wordstat permet de procéder à des analyses thématiques à partir de dictionnaires. Trois types d'études ont été menées selon cinq variables (journal, semaine, jour, mois, année) article par article: la fréquence des thèmes, leurs cooccurrences (analyses de liens et dendrogrammes selon un coefficient de force d'association) et leurs proximités. L'analyse est menée par le biais d'un dictionnaire construit sous forme de catégories que nous avions préétablies. Chaque catégorie regroupe le champ lexical du thème étudié. Nous en avons établi quatre :

- Économie

- Crise

- Union européenne

- National

Il faut noter que les deux dernières catégories admettent des sous-catégories afin d'affiner le codage. "Union européenne» admet quinze sous-thèmes et «national» en admet trois (annexe 1). Plus précisément, la construction du champ lexical a, elle aussi, été menée sous Wordstat (par l'outil de Lemmatisation) à partir d'une liste de mots-clés que nous avions établie. Le corpus d'articles a été construit à partir de la base de données d'Eureka, accessible via le serveur de l'Université de Montréal. 2775 articles ont été analysés selon la répartition suivante :

\begin{tabular}{|c|c|c|}
\cline { 2 - 3 } \multicolumn{1}{c|}{} & $\mathbf{2 0 0 9}$ & $\mathbf{2 0 1 4}$ \\
\hline Le Monde & 679 & 864 \\
\hline Le Figaro & 686 & 546 \\
\hline
\end{tabular}




\section{Analyse}

La modélisation sous Wordstat met en lumière une évolution entre 2009 et 2014 du traitement médiatique des élections européennes à deux niveaux. D'une part, nous observons une européanisation de la cartographie des thématiques entre 2009 et 2014. D'autre part, nous constatons la mise en marche d'une européanisation verticale (Guiraudon 2002) de l'espace public français.

Une modification de la cartographie des thématiques entre 2009 et 2014

Après avoir mené une analyse identique sur nos deux périodes, nous observons une européanisation de la cartographie des thèmes sur l'échiquier médiatique en 2014.

Pour la campagne des européennes en 2009, une première analyse par dendrogramme (tableau 1), met en évidence deux logiques. D'une part, nous constatons que la cartographie des thématiques en 2009 est hétérogène et suit le modèle suivant: il y a une association des thématiques nationales («acteurs nationaux» / «partis politiques nationaux») avec le duo «terminologie» / «Élections européennes» par l'entremise de «campagne électorale». À ce bloc, s'ajoutent, d'une part, les thèmes «crises » / «économie » et d'autre part, les thèmes «institution» / «lieu ». Une analyse par dendrogramme permet non seulement d'identifier des liens d'association entre différentes thématiques, mais aussi de les hiérarchiser. Le schéma ci-dessous nous montre que les deux principales thématiques sont les «acteurs nationaux» et les "partis politiques nationaux ».

Tableau 1 : Dendogramme - 2009

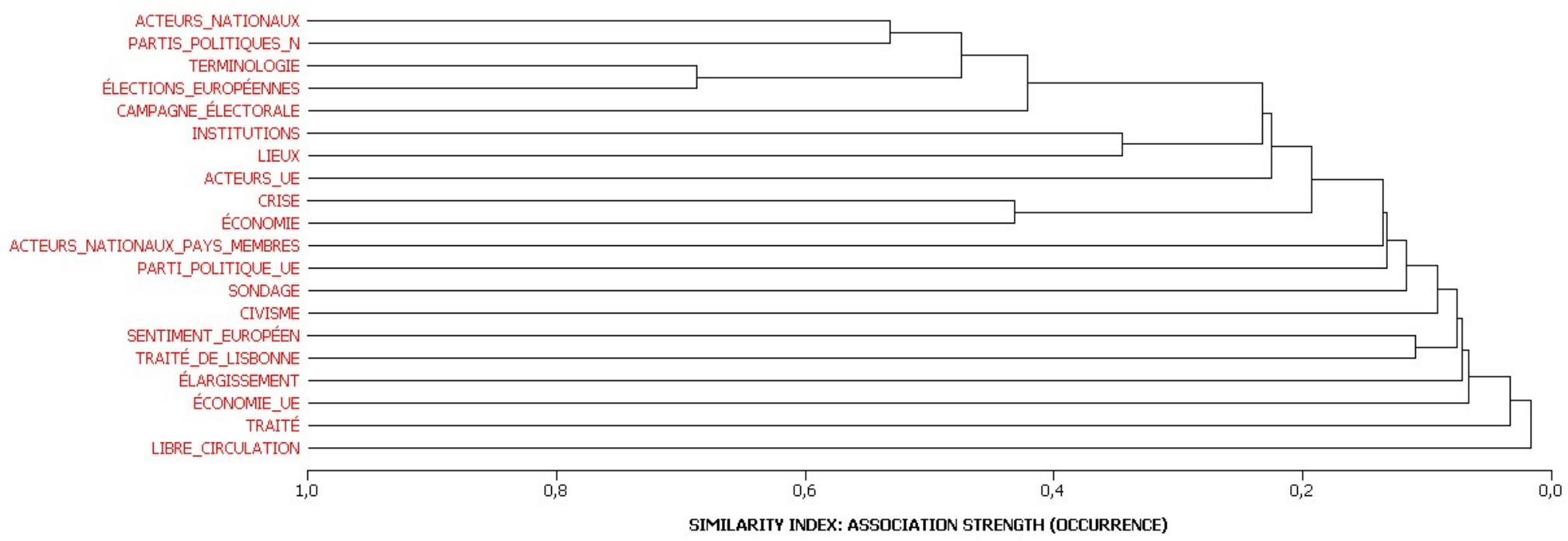


Les thématiques européennes n'apparaissent qu'en troisième et quatrième positions. En ce sens, ces deux logiques montrent que les élections européennes de 2009 sont portées par des acteurs nationaux, dans une arène politique exclusivement nationale et sur des thèmes nationaux. Même dans un «moment d'EPE », la portée des enjeux européens reste secondaire.

Une seconde analyse, par « analyse de lien », vient confirmer et compléter l'analyse par dendrogramme en mettant en évidence la «force », ou l'absence de force, de la relation entre les différentes thématiques. Quatre constats se dégagent de la modélisation (tableau 2) :

1. Il y a un lien significatif entre « acteurs nationaux » et « partis politiques nationaux »

2. Il y a un lien significatif entre «acteurs nationaux» et «élections européennes »

3. Il y a un lien significatif entre "partis politiques nationaux » et « élections européennes »

4. Les thématiques proprement européennes («politique_UE » et « acteurs_UE ») restent isolées du reste du schéma.

Tableau 2 : Analyse de liens - 2009

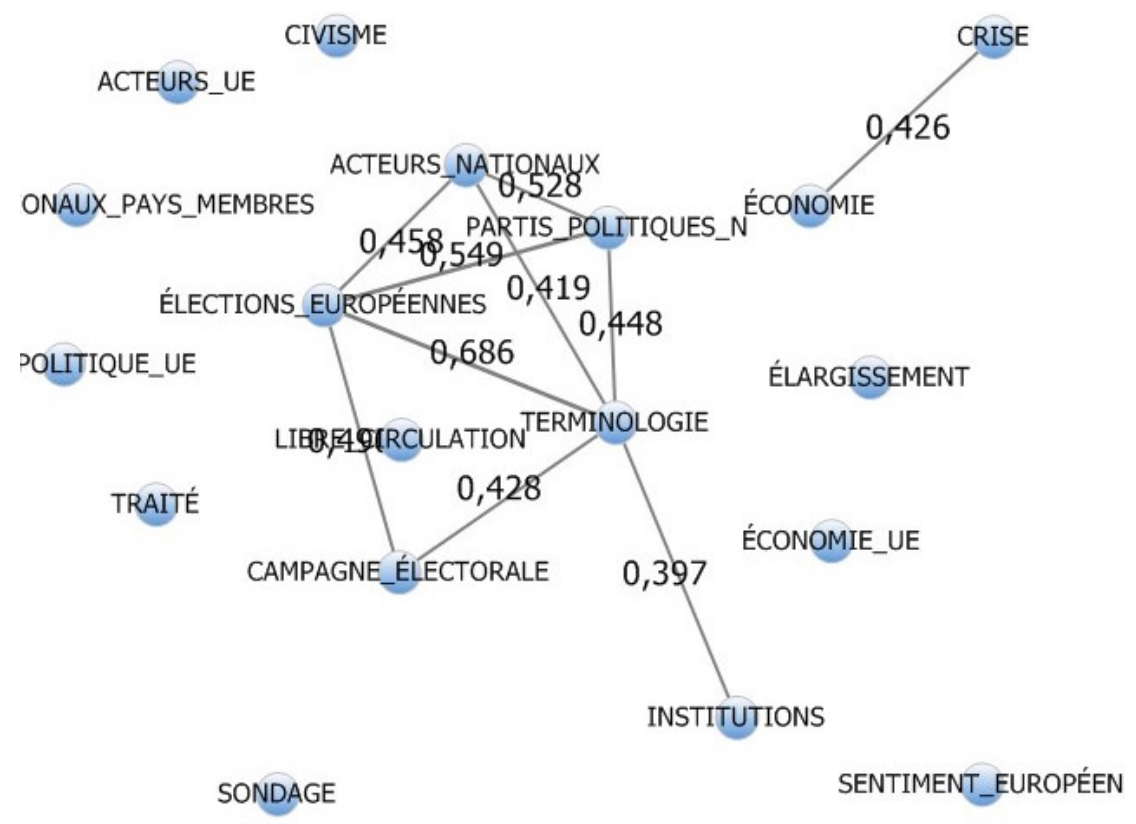


Suivant ces deux types de modélisation, un pattern spécifique se détache pour expliquer le traitement médiatique de la campagne des élections européennes de 2009. D'une part, la préséance des thématiques nationales - malgré le contexte de politique européenne - dans les médias français montre que la campagne des élections européennes s'est circonscrite à l'arène politique nationale. Union européenne et enjeux européens restent des acteurs secondaires dans un moment où ceux-ci devraient être au premier plan. D'autre part, le traitement médiatique, qu'il soit de centre gauche ou de droite, ne se détache pas du cadre national. La force des liens entre les thématiques nationales et l'absence de lien avec les thématiques européennes montrent que celles-ci demeurent un prétexte électoral de politique nationale. Ainsi, en 2009, même en temps de "moments européens ", l'UE ne semble pas bénéficier pas de sa propre arène politique au national et de son propre espace public en France.

Concernant la campagne des élections européennes de 2014, l'analyse par dendrogramme révèle que la hiérarchie des thématiques reste similaire avec «acteurs nationaux» et "partis politiques nationaux» en première position. Cependant, le regroupement des thématiques par force d'association s'avère différent et plus complexe (tableau 3). Trois groupes se dessinent :

1. Groupe 1 : Les thématiques nationales (« acteurs_nationaux»; «partis politiques nationaux ») sont associées à la thématique « campagne électorale ».

2. Groupe $2:$ La «crise » et les thématiques européennes («terminologie »; « élections européennes »; " économie UE » forment une nouvelle entité en relation directe avec le groupe 1.

3. Groupe 3: Les institutions européennes et les lieux européens sont directement associés avec le groupe 2 et indirectement avec le groupe 1. 
Tableau 3 : Dendrogramme - 2014

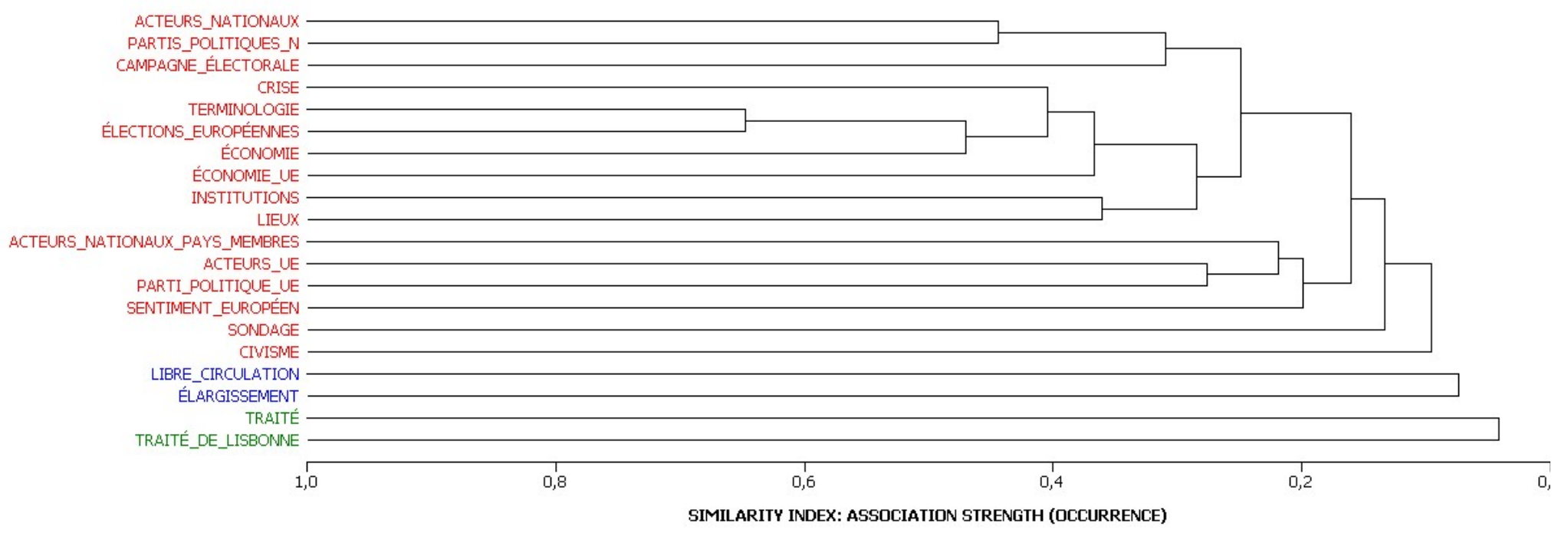

L'analyse de lien vient confirmer cette tendance : la répartition géographique des thématiques n'est plus centralisée autour des thématiques nationales, mais davantage dispersée (Tableau 4). Nous y voyons un isolement des «acteurs nationaux » et des " partis politiques nationaux » au profit d'une recentralisation autour des thématiques liées à l'économie (surtout la crise) et à l’UE.

Tableau 4 : Analyse de liens - 2014

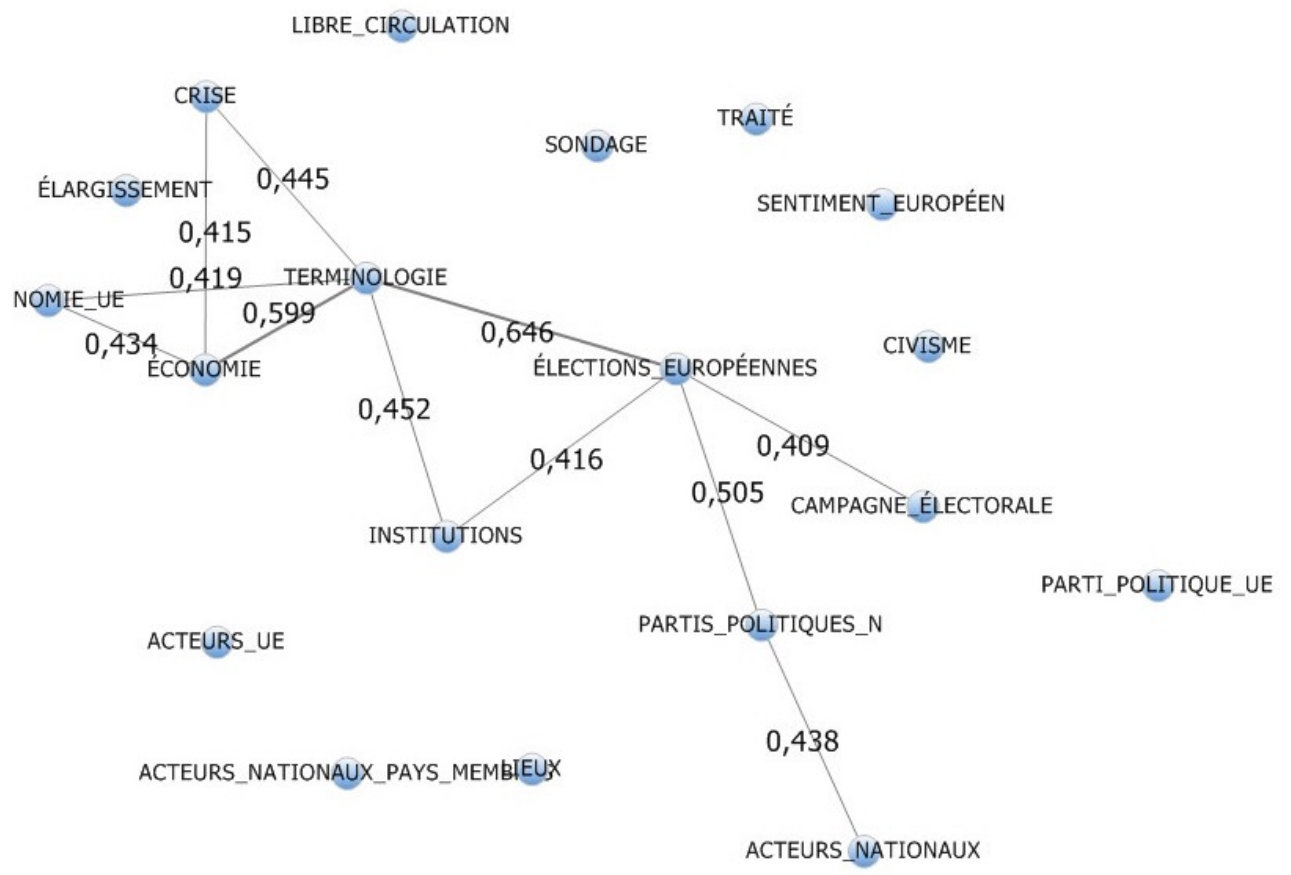


Suivant ces deux types de modélisation, le pattern établi dans la section précédente pour les élections de 2009 ne fonctionne pas pour les élections 2014. Le contexte de crise économique a modifié la cartographie des thématiques en réorganisant les associations entre les thèmes abordés par les médias. Le discours médiatique prend une dimension européenne qui était inexistante auparavant et que nous pouvons expliquer par deux facteurs :

- La proximité des thématiques «crise », «économie », « institutions » et «terminologie» montrent que les médias attribuent les enjeux économiques à l'arène politique européenne et non plus au niveau national.

- La force des liens entre les thématiques européennes et électorales corrélée à l'isolement des thématiques nationales montrent qu'un processus d'européanisation a débuté : les médias n'utilisent plus un cadrage national pour traiter des élections européennes, celles-ci bénéficient d'un traitement journalistique européen et à part entière.

En somme, les deux types de modélisations que nous avons menées (analyse de lien et dendrogramme), successivement en 2009 et 2014, suggèrent un effet de la crise économique dans le processus d'européanisation de l'espace public national français. Il s'agit maintenant de vérifier si les acteurs européens ont véritablement joué un rôle dans les débats de 2014.

\section{Une européanisation verticale de l'espace public français}

Au-delà d'une modification de la cartographie des thématiques sur l'échiquier médiatique, la modélisation avec Wordstat nous permet de qualifier ce processus «d'européanisation verticale» comme l'entend Virginie Guiraudon (Guiraudon 2002). Comme il a été dit dans le cadre théorique, cette forme d'européanisation se caractérise par l'importation d'acteurs et de questions européennes dans la sphère publique nationale: "Soit parce que des acteurs européens interviennent [proposition 1], soit parce que des acteurs nationaux interpellent les institutions européennes [proposition 2], ou se mobilisent au niveau européen, soit parce qu'un cadrage européen est utilisé pour justifier une 
position [proposition 3] ». Notre analyse permet de justifier empiriquement les propositions 1 et 3 .

"Des acteurs européens interviennent dans la sphère publique nationale »

Pour ce faire, nous sommes allées observer la présence des acteurs (chefs d'États et de gouvernements) des autres pays membres de l'UE dans la couverture médiatique de la campagne électorale. Par une analyse de liens, nous remarquons qu'en 2009, la catégorie «acteurs nationaux pays membres » n'a pas de valeur significative effective, la force des liens restant faible. Cependant, en 2014, la dynamique évolue. Les liens d'association sont plus forts et surtout, sont associés à de nouvelles thématiques. Nous observons cinq thématiques associées, toutes exclusivement européennes (tableau 5):

- «lieux»

- «parti politique UE»

- « institutions »

- « élections européennes »

- «terminologie»

De ces résultats, nous pouvons déduire que les acteurs des autres pays membres interviennent dans la couverture médiatique des élections européennes françaises, leur donnant une dimension transnationale. Il faut mentionner que 2014 est plus propice à cette transnationalisation des acteurs que 2009 : en effet, le processus des Spitzenkandidaten, où chaque parti européen a pu nommer un candidat à la présidence de la Commission européenne, a encouragé les candidats à mener des campagnes pan-européennes. Les principaux chefs de partis - allemands, luxembourgeois, belges ou grecs - sont ainsi intervenus dans la campagne française à travers les médias.

Toutefois, il est certain qu'il faut être prudent avec les interprétations, car la force des liens ne permet pas d'affirmer catégoriquement une telle association. Nous pouvons seulement souligner une évolution. Entre 2009 et 2014, une tendance semble s'installer. 
40 L'Union européenne et le sentiment d'appartenance

Tableau 5 : Proximité des thèmes avec la catégorie acteurs nationaux pays membres -2009

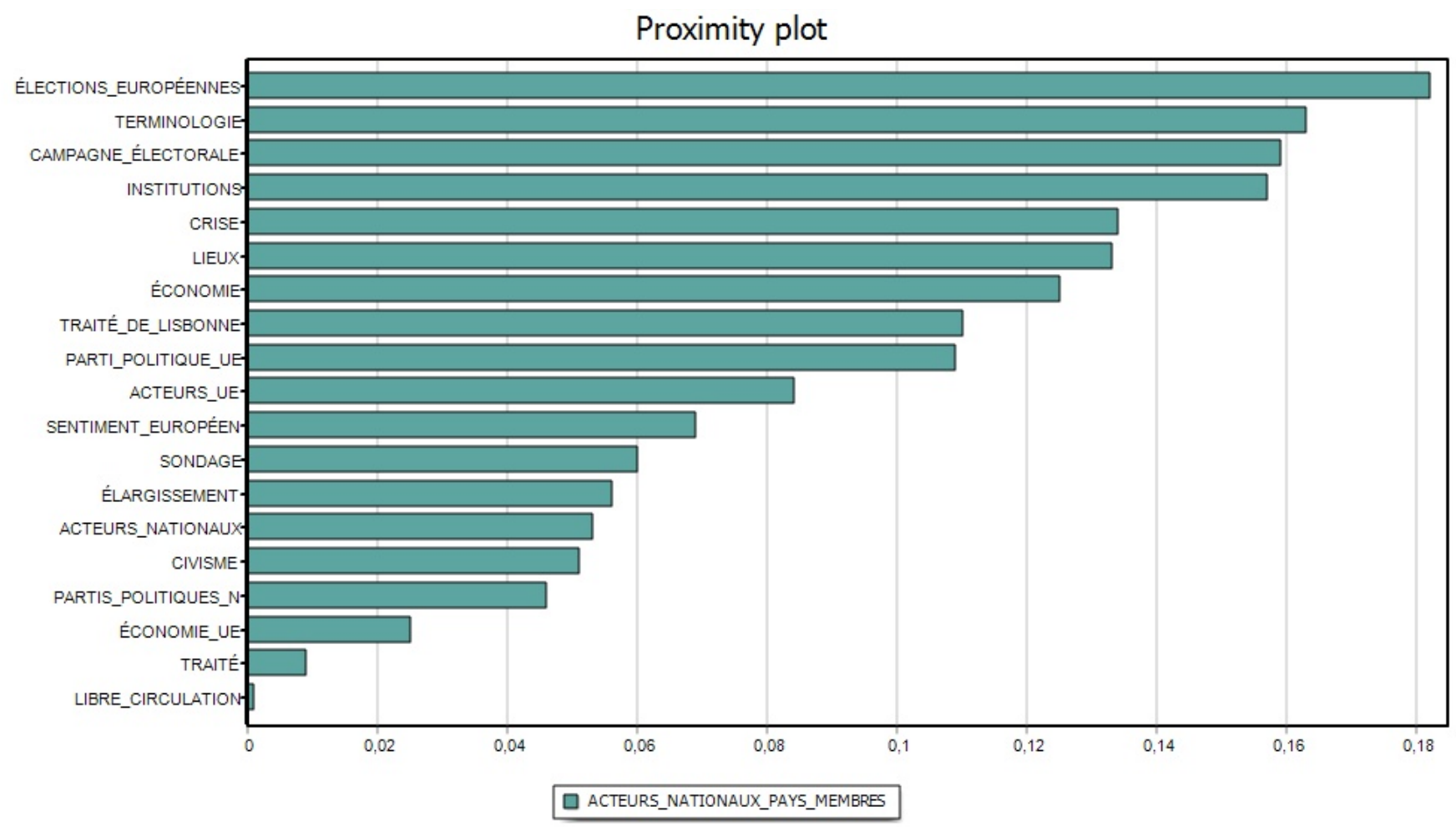

Tableau 6 : Proximité des thèmes avec la catégorie acteurs nationaux pays membres - 2014 Proximity plot

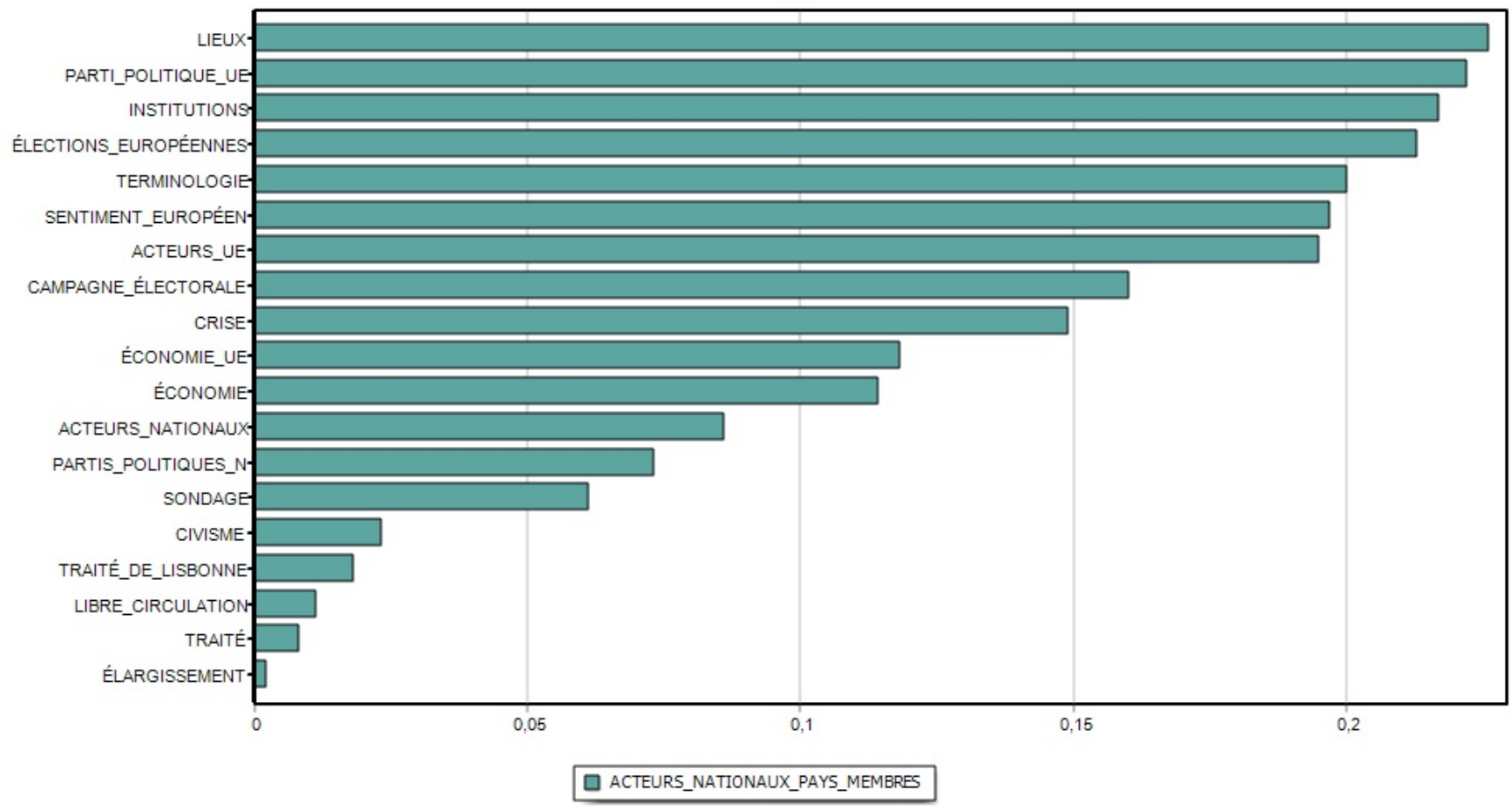




\section{Conclusion}

Dans cet article nous voulions tester si la crise économique avait un impact sur l'européanisation de l'espace public français. En menant une analyse de contenu, nous avons comparé la couverture médiatique de la campagne des élections européennes de 2009 et 2014. L'analyse de nos données suggère que la crise économique induit une européanisation de l'espace public français selon deux vecteurs : une modification de la cartographie des thématiques entre 2009 et 2014 et la mise en marche d'une européanisation verticale. Ainsi, par l'entremise d'une arène de communication dans laquelle les gouvernants et les gouvernés se regroupent et expriment des intérêts particuliers, des préoccupations ou des attentes qui influencent la prise de décision politique, nous apportons des éléments empiriques tangibles à la thèse selon laquelle les crises créent des espaces démocratiques au niveau européen.

Il est certain que notre recherche, de nature exploratoire et non définitive, comporte un certain nombre de limites. Tout d'abord, la causalité est impossible à établir entre la crise et le développement de l'espace public européen ; tout au plus pouvons-nous suggérer une corrélation qui s'explique de manière raisonnable par la mise en lumière des politiques européennes et le rôle croissant des acteurs européens au niveau national. Il existe en outre un biais de sélection des cas puisque la barrière de la langue restreint considérablement le choix de nos pays. Il serait intéressant de pouvoir mener l'analyse sur la totalité des pays de la zone euro. Même si l'analyse de contenu s'avère être outil pertinent, il ne faut pas oublier qu'elle induit aussi des biais. Nous pouvons voir deux principales limites de cette méthodologie: l'analyse de contenu médiatique induit une cueillette de données conditionnée à un accès aux archives médiatiques et une catégorisation qui force le chercheur à faire des choix.

\section{Bibliographie}

Albo, G., L. Panitch et V. Chibber (2013). Quelle stratégie? Résurgence des mouvements sociaux, combativité et politique. Saint-Joseph-du-Lac, M Éditeur. 
Baglioni, S. et A. Hurrelmann (2014). « The Politicization of European Integration in the Eurozone Crisis: Accounting for the Lack of Supranational Citizen Mobilization », Workshop on The Politicization of European Governance. Bremen, University of Bremen, 4-5 juillet 2014.

Bärenreuter, C., C. Brüll, M. Mokre et K. Wahl-Jorgensen (2009). " Overview of Research on the European Public Sphere », Eurosphere, working Paper ${ }^{\circ} 3$.

Brack, N., Y.-S. Rittelmeyer et C. Stanculescu. (2010). «L'Europe en campagne électorale : une analyse croisée des médias nationaux », Politique européenne 31, pp. 173-203.

Dacheux, E. (2003). L'Europe qui se construit : réflexions sur l'espace public européen. Saint-Étienne, Publications de l'Université de Saint-Etienne.

Dahlgren, P. et M. Relieu (2000). «L'espace public et l'internet. Structure, espace et communication », Réseaux 18(100), pp. 157-86.

De Vreese, Claes H., Susan A. Banducci, Holli A. Semetko, et Hajo G. Boomgaarden (2006). "The News Coverage of the 2004 European Parliamentary Election Campaign in 25 Countries », European Union Politics 7(4), pp. 477-504.

Desroches, D. et D. Innerarity (2011). Penser le temps politique. Entretiens philosophiques et contretemps avec Daniel Innerarity. Québec, Presses de l’Université Laval.

Frank, R. (2010). Building a European Public Sphere: From the 1950s to the Present = Un espace public européen en construction: des années 1950 à nos jours. Berlin, Peter Lang.

Freund, J. (1976). "Sur deux catégories de la dynamique polémogène », Communications 25(1), pp. 101-12.

Guiraudon, V. (2002). La place de l'Europe dans les débats nationaux: enjeux théoriques, protocole méthodologique et premiers résultats empiriques à partir du cas français, L'institutionnalisation de l'Europe, table ronde n. 5, Lille, 1821 septembre 2002.

Habermas, J. (1978). L'espace public. Paris, Éditions Payot.

Hix, S. (1999). The Political System of the European Union. New York, St. Martin's Press.

Hutter, S. Grande, E. et H.P. Kriesi. (2016). Politicising Europe: Integration and Mass Politics. Cambridge, Cambridge University Press. 
Koopmans, R. et P. Statham (2010). The Making of a European Public Sphere, Media Discourse and Political Contention. Cambridge, Cambridge University Press.

Liberti, F. (2012). "L'Union européenne et la crise de la dette», Revue internationale et stratégique 2(86), pp. 16-25.

Meijers, M. (2013). « The Euro-crisis as a Catalyst of the Europeanization of Public Spheres? A Cross-temporal Study of the Netherlands and Germany », LSE Europe In Question Series (LEQS), paper $n^{\circ} 62$.

Mercier, A. (2003). Vers un espace public européen ? Recherches sur l'Europe en construction. Paris, L'Harmattan.

OFCE, ECLM, IMK. 2012. «Independent Annual Growth Survey: Failed austerity in Europe, the way out». En ligne: https://www.iagsproject.org/documents/iags_report2013.pdf (page consultée le 11 mars 2016).

Olsen, J. P. (2002). « The Many Faces of Europeanization ». Journal of Common Market Studies 40(5), pp. 921-52.

Peter, J. et C.H. de Vreese (2004). «In Search of Europe: A Cross-National Comparative Study of the European Union in National Television News », The International Journal of Press/Politics 9(4), pp. 3-24.

Pfetsch, B. (2004). "The Voice of the Media in European Public Sphere: Comparative Analysis of Newspaper Editorials ». En ligne: http: / / europub.wzb.eu/Data/ reports / WP3 / D34\%20WP3\%20Integrated\%20Report.pdf (page consultée le 11 mars 2016).

Picard, R. G. (2015). The Euro Crisis in the Media: Journalistic Coverage of Economic Crisis and European Institutions. Londres, I.B.Tauris \& Co Ltd.

Trenz, H.-J. et K. Eder. (2004). «The Democratizing Dynamics of a European Public Sphere. Towards a Theory of Democratic Functionalism », European Journal of Social Theory 7(1), pp. 5-25.

Weisbein, J. (2002). La question de l'espace public européen. Paris, Presses de Sciences Po (P.F.N.S.P.) 
44 L'Union européenne et le sentiment d'appartenance

\section{Annexe}

\begin{tabular}{|c|c|c|}
\hline CATÉGORIE & $\begin{array}{l}\text { SOUS- } \\
\text { CATÉGORIE }\end{array}$ & ITEM \\
\hline Économie & & 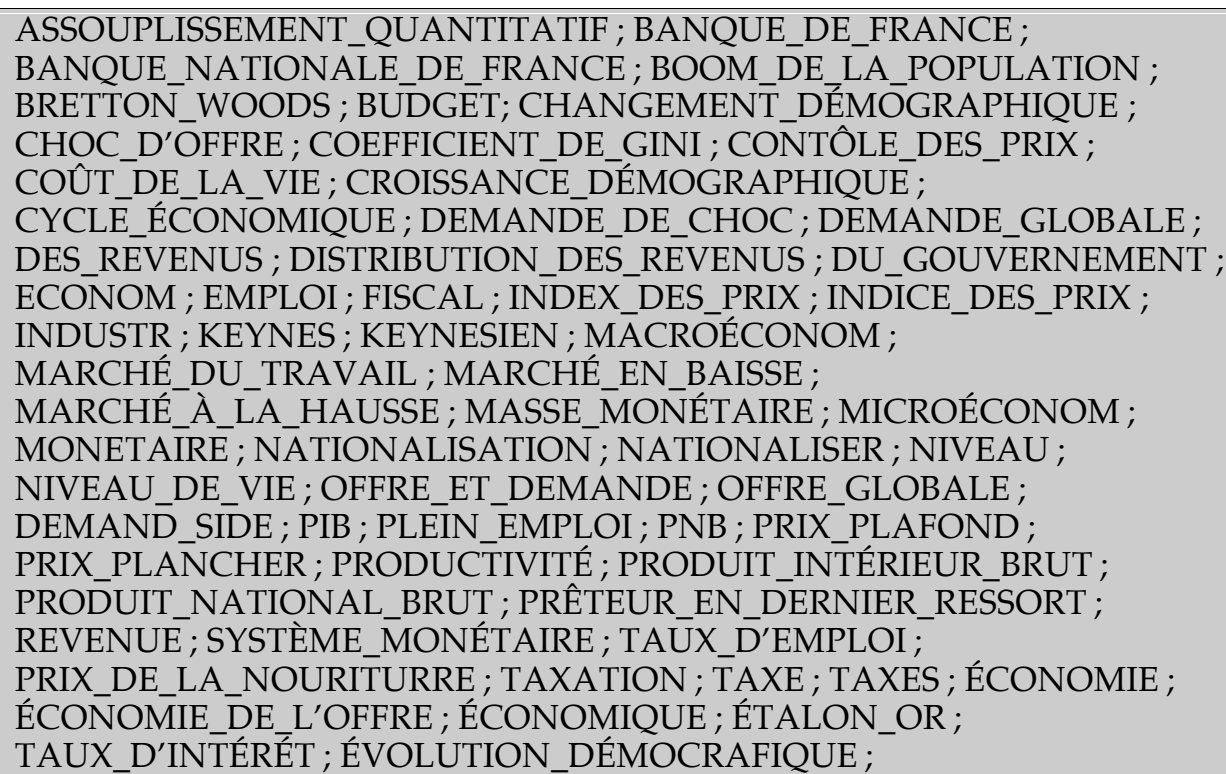 \\
\hline CRISE & & $\begin{array}{l}\text { AGENCES_DE_NOTATION ; AGENCE_DE_NOTATION ; AUSTÉRITÉ ; } \\
\text { CHOMAGE ; CONFLICT; CONFLIT ; CRISE;CRISE_ZONE_EURO ; } \\
\text { DEFICIT ; DETTE ; DÉFAUT_DE_CRÉDIT ; DÉFAUT_DE_PAIEMENT ; } \\
\text { DÉFLATION ; INFLATION; MARCHÉS_FINANCIERS ; } \\
\text { MARCHÉ_FINANCIER; PACTE_DE_STABILITÉ ; POOR'S; PRIVATIS ; } \\
\text { RIGUEUR ; RIGUEUR_BUDGETAIRE; SANS_EMPLOI ; SAUVETAGE ; } \\
\text { RECESSION ; SAUVETAGE_FINANCIER ; }\end{array}$ \\
\hline \multirow[t]{4}{*}{$\begin{array}{l}\text { UNION } \\
\text { EUROPÉENNE }\end{array}$} & CAMPAGNE_UE & $\begin{array}{l}\text { CAMPAGNE ; CANDIDAT ; CHEFS_DE_PARTI ; CHEF_DE_PARTI ; } \\
\text { COURSES_ÉLECTORALES ; COURSE_ÉLECTORALE; LEADER; } \\
\text { PROGRAMMES_ÉLECTORAUX; PROGRAMME_ÉLECTORALE; } \\
\text { THEMES_DE_CAMPAGNE ; THĖME_DE_CAMPAGNE ; }\end{array}$ \\
\hline & $\begin{array}{l}\text { PARTI_POLITIQU } \\
\text { E_UE }\end{array}$ & $\begin{array}{l}\text { EUROPE_ECOLOGIE; ACRE; ALDE; } \\
\text { ALLIANCE_DES_CONSERVATEURS_ET_RÉFORMISTES_EUROPÉENS ; } \\
\text { ALLIANCE_DES_LIBERAUX_ET_DES_DÉMOCRATES_POUR_L'EUROPE ; } \\
\text { DÉMOCRATES_LIBÉRAUX ; ECR; ELDD ; } \\
\text { EUROPE_DE_LA_LIBERTE_ET_DE_LA_DÉMOCRATIE_DIRECTE ; } \\
\text { GAUCHE_RADICALE; INDÉPENDANCE-DÉMOCRATIE ; } \\
\text { PARTI_DE_LA_GAUCHE_EUROPÉENNE ; } \\
\text { PARTI_POPULAIRE_EUROPÉEN ; } \\
\text { PARTI_VERT_EUROPÉEN; PGE ; PPE ; PARTI_SOCIALISTE_EUROPÉEN ; } \\
\text { PSE ; PVE ;SD;SOCIO_DÉMOCRATE; UKIP; } \\
\text { UNION_POUR_L'EUROPE_DES_NATIONS; UEN; VERT_ALE; }\end{array}$ \\
\hline & ACTEURS_UE & $\begin{array}{l}\text { BARROSO; BOVE; BUZEK; COHN_BENDIT; DAUL; } \\
\text { DRAGHI ; JUNCKER ; FONTELLO ; KAMALL ; KELLER ; } \\
\text { RASMUSSEN ; SCHULZ; TRICHET; STANICHEV ; TSIPRAS ; } \\
\text { TSÍPRAS ; TUSK ; VAN_ROMPUY ; VERHOFSTADT ; WATSON ; }\end{array}$ \\
\hline & CIVISME & $\begin{array}{l}\text { ABSTENIR ; ABSTENTIONNISME ; ABSTENUS ; ABSTIENDRA ; } \\
\text { ABSTIENNENT; ; ACCĖS_A_L'INFORMATION ; ALLER_VOTER; } \\
\text { CITOYENNETÉ_EUROPÉENNE; ABSTIENT ; CIVIQUE;CIVISME ; } \\
\text { DROITS_CIVILS ; DROIT_DE_VOTE ; } \\
\text { INSCRIPTION_SUR_LES_LISTES_ELECTORALES ; } \\
\text { LIBERTÉS_CIVILES ; LIBERTÉ_CIVILE ; VOTER ; }\end{array}$ \\
\hline
\end{tabular}




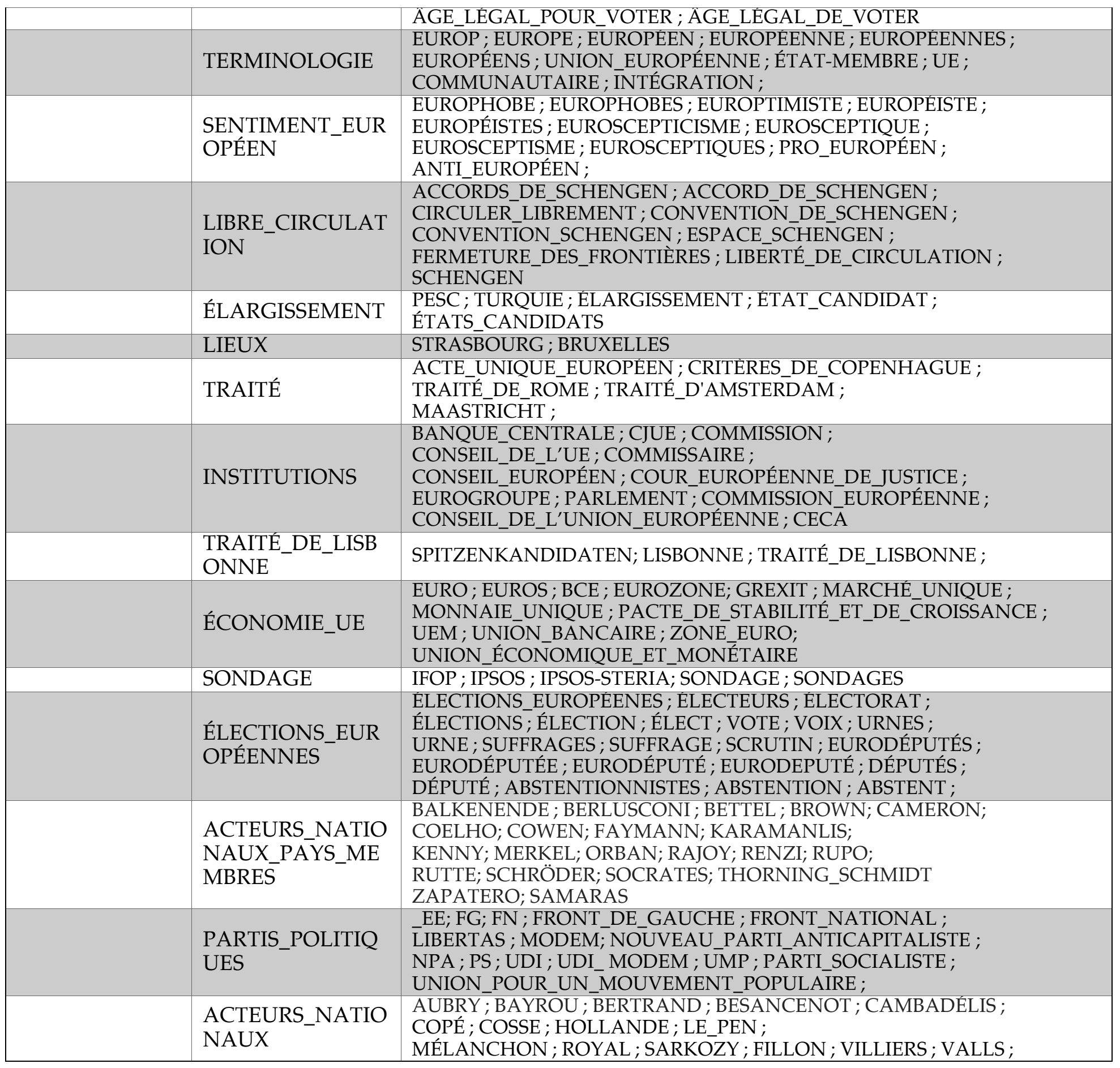

